\title{
THE EMPIRICAL DETERMINATION OF THE DAMPING CONSTANTS OF FRAUNHOFER LINES
}

\author{
C. DE JAGER ${ }^{1}$ and L. NEVEN ${ }^{2}$ \\ ${ }^{1}$ University Observatory, "Sonnenborgh", Utrecht, Netherlands \\ ${ }^{2}$ Royal Belgian Observatory, Uccle-Brussels, Belgium
}

(Received 10 May 1965)

\begin{abstract}
A systematic method is developed to determine the damping constant, $\gamma$, and its variation with optical depth, $\tau$, in the solar photosphere, using the observed profiles of wings of Fraunhofer lines and their center-to-limb variation.

The method is applied to the $3 s^{3} P^{\circ}-3 p^{3} D$ multiplet of carbon at $10,700 \AA$.

The resulting $\gamma(\tau)$ curve with its mean errors is shown in Fig. 2. This result can be explained by the usual assumption $\gamma(\tau)=\gamma_{\mathrm{H}, \mathrm{HA}}(\tau)+\gamma_{\mathrm{e}}(\tau)$, where $\gamma_{\mathrm{H}, \mathrm{He}}$ is the contribution to the damping constant by collisional broadening by neutral particles; $\gamma_{e}$ is due to the quadratic Stark effect of passing electrons. The "observed" values of the function $\gamma_{\mathrm{H}, \mathrm{He}}(\tau)$ agree perfectly with theoretical predictions. No theoretical predictions can yet be made for $\gamma_{e}(\tau)$ but the empirically derived values for this function are similar to values found from laboratory measurements for some lines of other elements, and look acceptable.
\end{abstract}

\section{INTRODUCTION}

Relatively little attention has been given to the determination of the damping constants, $\gamma$, of Fraunhofer lines. The first determination of the damping constants in the sun, by Minnaert and Mulders, ${ }^{(1)}$ showed $\gamma$ to be ten to a hundred times larger than the classical value. This large value had to be ascribed to collisional damping, either by collisions with electrons, or with neutral hydrogen. Unlike early-type stars, giant stars or the solar chromosphere, radiation damping is unimportant in spectra of the solar photosphere. Around 1940 several theories on the damping constants were developed which allow us to predict $\gamma$ in a semi-quantitative way. However, an exact theoretical computation of $\gamma$ presents, for many atoms, severe difficulties of a quantum-mechanical nature. For this reason an empirical determination of $\gamma$ and of its variation with depth in the solar photosphere appears to be important. That up to now such a determination has been made but rarely, ${ }^{(2,3)}$ can only partly be due to the high observational accuracy required. It appears that the difficulties involved in separating the influence of the damping constant on the line profile from the influence of the model of the photosphere have been overestimated.

In this paper we give a simple systematic method for determining $\gamma$. The method is applied to the $3 s^{3} P^{\circ}-3 p^{3} D$ multiplet of carbon near $10,700 \AA$. We have taken this multiplet since we have already used it as a test example for the development of a systematic method for investigating Fraunhofer line profiles. Previously we showed already how to determine, empirically, four of the parameters that influence the line profiles: 
the convective velocity component, $v_{c},{ }^{(4)}$

the abundance, $A_{C},{ }^{(5)}$

In this paper we will complete the series by describing a method for determining the damping constant $\gamma$. We intend to publish a descriptive review of our systematic method for analyzing line profiles. ${ }^{(6)}$ We have furthermore started applying this method to a large number of solar line profiles that have been acquired during the last few years. ${ }^{(7)}$

With the five unknowns thus found, one should be able to compute the whole profile of the spectral lines and to compare it with the observations. This procedure would make possible a final checking of the results.

\section{THE ABSORPTION COEFFICIENT IN THE WINGS OF} FRAUNHOFER LINES

In well-known notation, the absorption coefficient $\kappa(v)$ at a wavelength $v=\Delta \lambda / \Delta \lambda_{D}$ in the region of a Fraunhofer line is given by

$$
\kappa(v)=\kappa_{c} \mathrm{H}(\alpha, v)
$$

where $\kappa_{c}$ is the absorption coefficient in the line center $/ g$. For large values of $v$, and for $\alpha \ll 1$ :

Further

$$
\mathrm{H}(\alpha, v)=\frac{\alpha}{v^{2}(\pi)^{\frac{1}{2}}} *
$$

$$
\begin{aligned}
\alpha & =\frac{\gamma}{2 \Delta \omega_{D}}=\frac{\gamma \lambda^{2}}{4 \pi c \Delta \lambda_{D}}, \\
v & =\Delta \lambda / \Delta \lambda_{D} \\
\kappa_{c} & =\frac{(\pi)^{\frac{1}{2}} e^{2}}{m c^{2}} \frac{\lambda^{2} \mathfrak{N}}{\Delta \lambda_{D}}\left[1-\exp \left(-c_{2} / \lambda T\right)\right] \\
& =\frac{(\pi)^{\frac{1}{2}} e^{2} \lambda^{2}}{m c^{2} \Delta \lambda_{D}} f g A_{e l} \frac{b_{r . s}}{g} \frac{B}{4+B} \frac{1}{m_{\mathrm{H}}}\left[1-\exp \left(-c_{2} / \lambda T\right)\right]
\end{aligned}
$$

where $\mathfrak{N}$ is the number of classical oscillators per gram, $A_{e l}$ is the abundance of the element $=N_{e l} / N_{\mathrm{H}}, b_{r, s}$ is the fraction of the atoms in the relevant ionization and excitation level, $B=N_{\mathrm{H}} / N_{\mathrm{He}}, m_{\mathrm{H}}$ is the mass of the hydrogen atom.

The other symbols have their usual meaning.

Combining equations (1)-(5), one obtains

where

$$
\kappa(\Delta \lambda)=C_{1} \lambda^{4} \frac{\gamma f g A_{e l}}{\Delta \lambda^{2}} \frac{b_{r, s}}{g}\left[1-\exp \left(-c_{2} / \lambda T\right)\right]
$$

$$
C_{1}=\frac{e^{2}}{4 \pi m c^{3}} \frac{B}{4+B} \frac{1}{m_{\mathrm{H}}}=0 \cdot 260,
$$

* This expression is based on the assumption of symmetrical broadening without appreciable line shift. This assumption is confirmed by the observations, which show the lines to be symmetric, at least at the large distances to the line center to which the present discussion refers. 
if we assume $B=5 \cdot 5$ (Unsöld mixture).

If we further introduce the "wing-parameter"

$$
V=0 \cdot 260 \frac{\gamma f g A_{e l}}{\Delta \lambda^{2}}
$$

equation (6) becomes

$$
\kappa(V)=\lambda^{4} \frac{b_{r, s}}{g}\left[1-\exp \left(c_{2} / \lambda T\right)\right] . V
$$

This formula is applicable to all spectral lines provided that one confines oneself to that part of the line profile where the wing approximation $\kappa \propto \Delta \lambda^{-2}$ is correct.

\section{THE EMPIRICAL DETERMINATION OF $\gamma\left(\tau_{0}\right)$}

For an assumed value of $V$ and for a given model of the photosphere, one can easily compute the corresponding selective optical depth by numerical integration:

$$
\tau(V)=\int_{0}^{\tau_{0}} \frac{\kappa(V)}{\kappa_{0}} \mathrm{~d} \tau_{0},
$$

where $\kappa_{0}$ and $\tau_{0}$ are the absorption coefficient and optical depth for $\lambda=5000 \AA$. Similarly, one finds the continuous optical depth $\tau_{\lambda}$ for the continuous spectrum at $10700 \AA ̊$. With

and

$$
\kappa=\kappa_{\lambda}+\kappa(V),
$$

$$
\tau=\tau_{\lambda}+\tau(V),
$$

one finds the relative line depression $d(V)$ with

$$
d(V)=\frac{\int_{0}^{\infty} B\left(\tau_{0}\right) \exp \left(-\tau_{\lambda} \sec \theta\right) \sec \theta \mathrm{d} \tau_{\lambda}-\int_{0}^{\infty} B\left(\tau_{0}\right) \exp (-\tau \sec \theta) \sec \theta \mathrm{d} \tau}{\int_{0}^{\infty} B\left(\tau_{0}\right) \exp \left(-\tau_{\lambda} \sec \theta\right) \sec \theta \mathrm{d} \tau_{\lambda}} .
$$

In equation (9) we introduce $B$ instead of $S$ and, hence, assumed L.T.E. This assumption is validated by our finding ${ }^{(5)}$ that $S \neq B$ only for $\tau_{0}<0 \cdot 2$, and (as will be shown further on) that the depression $d$ is formed mainly in very deep layers, where $\tau_{0}>0.6$ (see the average $\tau_{0}$-values, denoted by $\tau_{0}^{*}$, and given in the last two lines of Table 1 ).

It is important to have an impression of the average depth of formation $\tau_{0}^{*}$ of the line depression, which may be found as follows:

If we set

$$
\Delta\left(\tau_{0}\right)=\frac{\kappa_{\lambda}}{\kappa_{0}} \exp \left(-\tau_{\lambda} \sec \theta\right)-\frac{\kappa}{\kappa_{0}} \exp (-\tau \sec \theta),
$$


TABle 1. $K, A_{C} f_{\gamma} \cdot \gamma^{*}$, The $\tau_{0}^{*}$ Range, AND the mean error $\mu_{\gamma}$ OF $\gamma^{*}$. The table Contains $\gamma^{*} \times 10^{-9}$

\begin{tabular}{|c|c|c|c|c|c|c|c|}
\hline & $\begin{array}{l}\cos \theta \\
\mathrm{K} \times 10^{24}\end{array}$ & $\begin{array}{l}1 \cdot 00 \\
2 \cdot 15\end{array}$ & $\begin{array}{l}0 \cdot 75 \\
2 \cdot 20\end{array}$ & $\begin{array}{l}0 \cdot 60 \\
2 \cdot 27\end{array}$ & $\begin{array}{l}0 \cdot 50 \\
2 \cdot 40\end{array}$ & $\begin{array}{l}0 \cdot 40 \\
2 \cdot 40\end{array}$ & $\begin{array}{l}0 \cdot 30 \\
1-80\end{array}$ \\
\hline$\lambda(\AA)$ & $A_{C} f_{\gamma}$ & & & & & & \\
\hline $\begin{array}{l}10691 \\
10683 \\
10685 \\
10730 \\
10707\end{array}$ & $\begin{array}{l}8.6 \times 10^{-4} \\
4.8 \\
3.3 \\
1.6 \\
1.6\end{array}$ & $\begin{array}{l}4 \cdot 52 \\
5 \cdot 40 \\
4 \cdot 23 \\
7 \cdot 45 \\
6 \cdot 31\end{array}$ & $\begin{array}{l}4 \cdot 02 \\
4 \cdot 96 \\
3 \cdot 72 \\
5 \cdot 76 \\
5 \cdot 30\end{array}$ & $\begin{array}{l}4 \cdot 12 \\
3 \cdot 41 \\
2 \cdot 59 \\
5 \cdot 56 \\
2 \cdot 57\end{array}$ & $\begin{array}{l}1 \cdot 98 \\
2 \cdot 95 \\
1 \cdot 90 \\
2 \cdot 84 \\
3 \cdot 24\end{array}$ & $\begin{array}{l}1 \cdot 98 \\
3 \cdot 64 \\
1 \cdot 69 \\
3 \cdot 66 \\
2 \cdot 42\end{array}$ & $\begin{array}{l}2 \cdot 20 \\
4 \cdot 10 \\
2 \cdot 74 \\
4 \cdot 32 \\
3 \cdot 88\end{array}$ \\
\hline & $\begin{array}{l}\gamma^{*} \times 10^{-9} \\
\mu_{\gamma} \times 10^{-9}\end{array}$ & $\begin{array}{l}5 \cdot 58 \\
0 \cdot 61\end{array}$ & $\begin{array}{l}4 \cdot 74 \\
0 \cdot 40\end{array}$ & $\begin{array}{l}3 \cdot 66 \\
0 \cdot 54\end{array}$ & $\begin{array}{l}2.59 \\
0.27\end{array}$ & $\begin{array}{l}2 \cdot 68 \\
0.40\end{array}$ & $\begin{array}{l}3 \cdot 45 \\
0 \cdot 42\end{array}$ \\
\hline & $\tau^{*}$-range & $\begin{array}{l}1.77 \\
1.96\end{array}$ & $\begin{array}{l}1.42 \\
1.53\end{array}$ & $\begin{array}{l}1 \cdot 18 \\
1 \cdot 26\end{array}$ & $\begin{array}{l}1.04 \\
1.08\end{array}$ & $\begin{array}{l}0.86 \\
0.90\end{array}$ & $\begin{array}{l}0.68 \\
0.71\end{array}$ \\
\hline
\end{tabular}

then

$$
\tau_{0}^{*}=\frac{\int_{0}^{\infty} \tau_{0} \Delta\left(\tau_{0}\right) \mathrm{d} \tau_{0}}{\int_{0}^{\infty} \Delta\left(\tau_{0}\right) \mathrm{d} \tau_{0}} .
$$

The procedure used in the investigation was as follows. First we computed the $d(V)$ and the $\tau_{0}^{*}(V)$ relations. The computations were made for the Utrecht Reference Model (1964) of the photosphere ${ }^{(8)}$ separately for each of the three columns of this model.

We chose a small value of $V$ and assumed a $\sec \theta$ value. For these values the corresponding value of the depression $d$ was computed with the IBM 1620 computer of the Uccle Observatory. If $d$ appeared to be smaller than $10^{-3}$, the computation was repeated for a new $V$-value that was three times larger than the previous one; if $d>10^{-3}$ the resulting values of $d$ and of $\tau_{0}^{*}$ were printed and the computations were repeated for a new value of $V$ which was 1.4 times larger than the previous value.

This procedurc was repcated until a value of $d$ was reached that exceeded 0.08 (arbitrarily chosen upper limit).

The computations were made for the three columns of the URP 1964 model, and for six values of $\cos \theta$, ranging from $0 \cdot 3$ to $1 \cdot 0$.

In Fig. 1 we show, as an example, the resulting values of $d$ and of $\tau_{0}^{*}$ as a function of $V$ for the three elements, and for $\cos \theta=1 \cdot 00$. The differences between $d\left(\tau_{0}^{*}\right)$ values for the three columns are small but by no means negligible; at certain $V$-values the ratio between the extreme values is a factor 2 . Since the relative contribution of the three kinds of photospheric columns is unknown we assumed them to be equally important and determined their straight mean value (solid curve) which we call $\bar{d}$. We do not show the results for other values of $\cos \theta$; these appeared to be ncarly identical with those found 


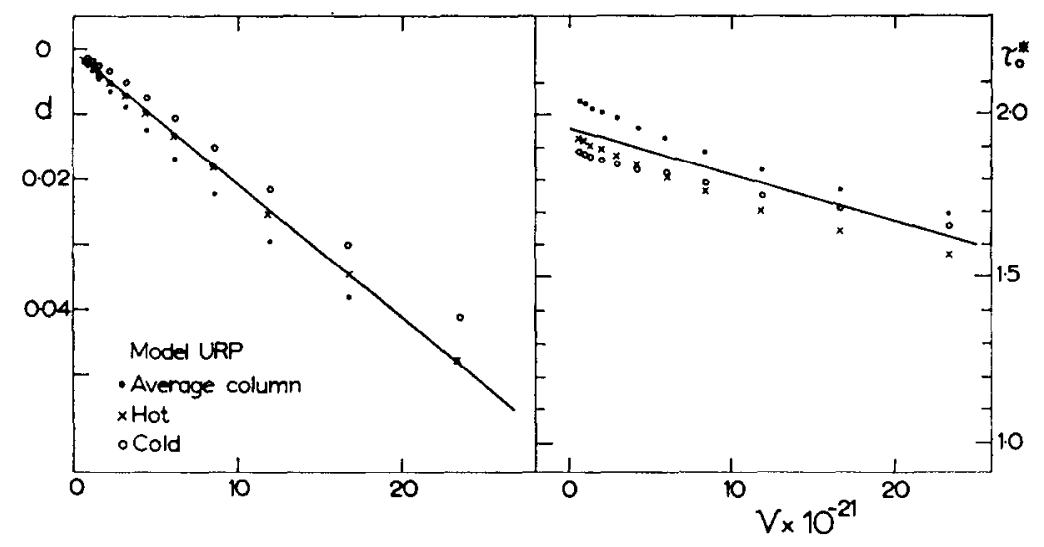

FIG. 1.

for the disc centre, at least for $d<0.05$. This is apparcntly duc to the fact that the gradient of $B\left(\tau_{\lambda}\right)$ is nearly constant in the relevant parts of the photosphere. For $\bar{d}$-values smaller than about 0.05 it appears possible to write $\bar{d}=K V$, where $K$ is a constant. For the various $\cos \theta$-values these $K$-values are given in the second line of Table 1 .

In order to determine $\gamma$, the following procedure was used. The observed depressions $d$ in the extreme line wings were represented by

$$
d_{\mathrm{obs}}=C(\Delta \lambda)^{-2} \text {. }
$$

We know further that

$$
\bar{d}=K V
$$

Here $C$ and $K$ are constants.

From the equations (11) and (12), setting $d_{\mathrm{obs}}=d$, we derive the expression

$$
V=\frac{C}{K(\Delta \lambda)^{2}} .
$$

Combining equation (13) with equation (7), one obtains

$$
A_{C} \gamma=C /(0 \cdot 260 f g K) \text {. }
$$

Here all right-hand member quantities are known so that $A_{C} \gamma$ may be found.

The $C$-values have already been given previously ${ }^{(4)}$. The values of $K$, derived from the computations described in this paper are given in the second line of Table 1 . In order to obtain an estimate of $\gamma$ we assumed $\log A_{C}=\overline{4} \cdot 61,{ }^{(6)}$ and so obtained the $A_{C} f g$ values given in the second column of Table 1. Further, the Table gives the resulting $\gamma$-values.

We also give for each $\cos \theta$ the average $\gamma$-value $\left(\gamma^{*}\right)$ and its mean error. Furthermore, we give the range of $\tau_{0}^{*}$ values to which the $\gamma^{*}$ values apply. This $\tau_{0}^{*}$-range was found from the computed $\tau_{0}^{*}(d)$ relation, and from the extreme $d$-values where the linear relations (11) and (12) are still applicable. 
The resulting $\gamma^{*}-\tau_{0}^{*}$ relation is shown in Fig. 2. It is clear that the mean error in $\gamma$ is still rather large; this is due to the uncertainties in the determination of $C$.



FIG. 2.

\section{COMPARISON BETWEEN THEORY AND OBSERVATIONS}

Although we want to postpone a thorough discussion of the results, when $\gamma$-determinations for a greater number of spectral lines are available, we will here draw attention to the shape of the $\gamma\left(\tau_{0}^{*}\right)$ curve which consists of a nearly horizontal part for $\tau_{0}^{*}<1$, followed by a part where $\gamma^{*}$ increases with depth. This latter part can easily be explained as being due to collisions with free electrons. For these collisions we have (ref. 8: eq. VII. 22, p. 66):

$$
\log \gamma_{e}=\log K+\log P_{e}+5 / 6 \log \Theta \approx \log K_{1}+\log P_{e} .
$$

Since $\log \Theta$ is nearly constant, $K_{1}$ is nearly constant too.

The $\gamma_{e}\left(\tau_{0}\right)$ gradient is virtually equal to the $\log P_{e}\left(\tau_{0}\right)$ gradient, which seems to apply to $\tau_{0}>1$.

In the less deep photospheric regions, where $\gamma$ seems nearly constant, the effect of collisions with neutral $\mathrm{H}$ and $\mathrm{He}$ particles seems to dominate. For these we have

$$
\log \gamma_{\mathrm{H}, \mathrm{He}}=\log K_{2}+\log P_{g},
$$

where $K_{2}$ is a nearly constant function of depth (ref. 8: eq. VII, 19 and VII, p. 20). Since also $P_{g}$ changes only slightly with depth, $\gamma_{H_{\text {,He }}}$ is also nearly constant with depth. A rapid empirical determination of the values of $K_{1}$ and $K_{2}$ is made as follows:

We take two representative parts of the photosphere. At $\tau_{0}=0 \cdot 7$, where $\gamma$ is still nearly constant, $\gamma=3 \times 10^{9}$. At $\tau=1 \cdot 85$, in the steeper part of the $\gamma\left(\tau_{0}\right)$ curve we have $\gamma=5.6 \times 10^{9}$. Since at these two depths $P_{e}=48$ and 220 , and $P_{g}=1.48 \times 10^{5}$ and 
$1.75-10^{5}$ respectively, we obtain two linear equations with the two unknowns $K_{1}$ and $K_{2}$. The solution is

$$
\begin{aligned}
& K_{1}=1.29 \times 10^{7}, \\
& K_{2}=1.61 \times 10^{4} .
\end{aligned}
$$

Next, these two values are compared with theoretical predictions. For collisions with neutral $\mathrm{H}$ and $\mathrm{He}$ we have ${ }^{(8)}$

$$
\begin{aligned}
\log \gamma_{\mathrm{H} . \mathrm{He}}=3 \cdot 84 & +\log P_{g}+0 \cdot 7 \log \Theta+\log \left(n_{0} / \Sigma n_{r}\right)_{\mathrm{H}} \\
& +\frac{2}{5} \log \left[\left(\frac{13 \cdot 6 Z}{\chi_{r}-\chi_{r . s}}\right)_{\text {upper }}^{2}-\left(\frac{13 \cdot 6 Z}{\chi_{\mathrm{r}}-\chi_{r, \mathrm{~s}}}\right)_{\text {lower }}^{2}\right]
\end{aligned}
$$

Since hydrogen is virtually neutral, $\log \left(n_{0} / \Sigma n_{r}\right)_{\mathrm{H}}=0$. Inserting $\Theta=0.75 ; \chi=11.20 \mathrm{eV}$; $x_{r, s}=7.45$ and $8.60 \mathrm{eV}$, we predict a theoretical value $K_{2}=1.62 \times 10^{4}$, which is in better agreement with the "observed" value than one could expect from the inaccuracy of the observed $\gamma$ values! This result proves that the observations confirm completely the theory on the contribution to the damping by collisions with neutral particles. There are no measurements or theories that would enable us to predict $K_{1}$ for the carbon lines studied by us. However, from the value of $K_{1}$ found above one can derive a value for the constant $K$ occurring in equation (15); it is

$$
\log K=7 \cdot 21 \text {. }
$$

This is a very acceptable value, which is completely in the range of other values for this constant, measured for other lines (ref. 8: Table VII, 3, p. 66).

We conclude that there is fair agreement between theory and observations on the damping of neutral carbon in the solar photosphere.

Acknowledgements - Our sincere thanks are due to Mr. W. NIJs, Uccle Observatory, who has performed the greater part of the numerical computations and who made all the numerical programming. We appreciate the valuable remarks made by Dr. A. SchadeE.

We also thank Mr. R. HENDRICXs, Brussels University, for effective help in some of the computations.

\section{REFERENCES}

1. M. G. J. MinnAert and G. F. J. Mulders, Z. Astrophys. 2, 165 (1932).

2. P. TEn Bruggencate and J. Houtgast, $Z$. Astrophys. 20, 149 (1940).

3. H. H. VoIGT, Z. Astrophys. 27, 82 (1949).

4. C. DE JAGER and L. Neven, Mem. Soc. Roy. Sci. Liège, 9, 213 (Liège Colloquium 1963) (1964).

5. C. DE JAGER and L. NEVEN, in The Abundance Determination in Stellar Spectra, Utrecht Symposium 1964 (1965).

6. C. DE JAGER and L. NEVEN, in preparation (1966).

7. C. DE JAGER and L. NEVEN, in preparation (1966).

8. C. DE JAGER and L. NEVEN, Rech. Obs. Astr. Utrecht, 13, (4) (1957).

9. J. R. W. Heintze, H. HubENET and C. DE JAGER, Bull. Astr. Insts. Netherlds. 17, 442 (1964). 